\title{
Ion beam analysis based on cellular nonlinear networks
}

\author{
V. Senger ${ }^{1}$, R. Tetzlaff ${ }^{1}$, H. Reichau ${ }^{2}$, and U. Ratzinger ${ }^{2}$ \\ ${ }^{1}$ Faculty of Electrical Eng. \& Information Technology, Dresden University of Technology, 01062 Dresden, Germany \\ ${ }^{2}$ Institute of Applied Physics, Goethe University Frankfurt, Max-von-Laue-Str. 1, 60438 Frankfurt, Germany
}

\begin{abstract}
The development of a non- destructive measurement method for ion beam parameters has been treated in various projects. Although results are promising, the high complexity of beam dynamics has made it impossible to implement a real time process control up to now. In this paper we will propose analysing methods based on the dynamics of Cellular Nonlinear Networks (CNN) that can be implemented on pixel parallel CNN based architectures and yield satisfying results even at low resolutions.
\end{abstract}

\section{Cellular Nonlinear Networks}

CNN were introduced 1988 by Chua and Yang in (Chua and Yang, 1988). A very general definition is given in (Chua and Roska, 1998) stating "a Cellular Nonlinear Network(CNN) is any spatial arrangement of locally coupled cells, where each cell is a dynamical system which has an input, an output and a state evolving according to some prescribed dynamical laws". Each CNN cell is directly connected to its neighbouring cells. Even though there are no restrictions regarding coupling laws and dynamics mentioned in the above definition, usually a translation invariant state equation known as the standard CNN equation is used. It is given by

$\dot{x}_{i, j}=-x_{i, j}+\sum_{k, l \in S_{i, j}} a_{k-i, l-j} y_{k, l}+\sum_{k, l \in S_{i, j}} b_{k-i, l-j} u_{k, l}+z$.

with $x_{i, j}$ denoting the state, $y_{i, j}$ the output, $z$ a constant bias and $u_{i, j}$ the input of the cell. $S_{i, j}$ is called the influence sphere of the cell. Its size is given by a neighbouring radius $r$ that denotes how many cells in each direction are directly connected to a given cell. The state of the cell is mapped onto the output through a nonlinear function $f(\cdot)$, which in

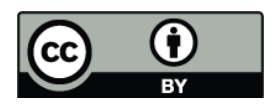

Correspondence to: V. Senger (vanessa.senger@tu-dresden.de) most cases is a piecewise linear function

$f\left(x_{i, j}\right)=y_{i, j}=\frac{1}{2}\left(\left|x_{i, j}+1\right|+\left|x_{i, j}-1\right|\right)$

called standard nonlinearity. A standard CNN with a neighbourhood radius of $r=1$ can be described by input and output weights and a bias $z$ which form a set of 19 parameters. Mainly, CNN have been intensively studied and applied in image processing problems leading to a huge variety of networks specified in the library (CSWC Lab, 2007).

\subsection{Hardware realizations of CNN}

The concept of CNN was proposed with special focus on circuit realizations - pixel parallel systems that are based on the so-called Full Range CNN equation (Chua and Roska, 1992) given by

$$
\begin{array}{r}
\dot{x}_{i, j}=-x_{i, j}-g\left(x_{i, j}(t)\right)+\sum_{|k| \leq r,|l| \leq r} \hat{A}_{k, l} \cdot y_{i+k, j+l} \\
+\sum_{|k| \leq r,|l| \leq r} \hat{B}_{k, l} \cdot u_{i+k, j+l}+\hat{I}
\end{array}
$$

with a function $g(x i, j(t))$ that limits the state of the CNN to an interval suitable for circuit realizations. During the last few years, various platforms based on FPGA architectures (Nagy and Szolgay, 2003) as well as on platforms based on analogue computing (Anafocus, 2005) have been proposed. In our project, we used a prototype version of an analogue system called EyeRIS v1.1 system (Anafocus, 2005). It consists essentially of two components: a NIOS II FPGAprocessor used for process control, programming, and input/output of data using a USB 1.1 connection, and an ACE 16k Focal Plane Processor (FPP) with $128 \times 128$ cells based on CNN. Additionally, each cell has some logic processors which can execute basic logic functions such as XOR and NAND as pixel parallel operations and an optical sensor, thus enabling the system to aquire and process images directly.

Published by Copernicus Publications on behalf of the URSI Landesausschuss in der Bundesrepublik Deutschland e.V. 


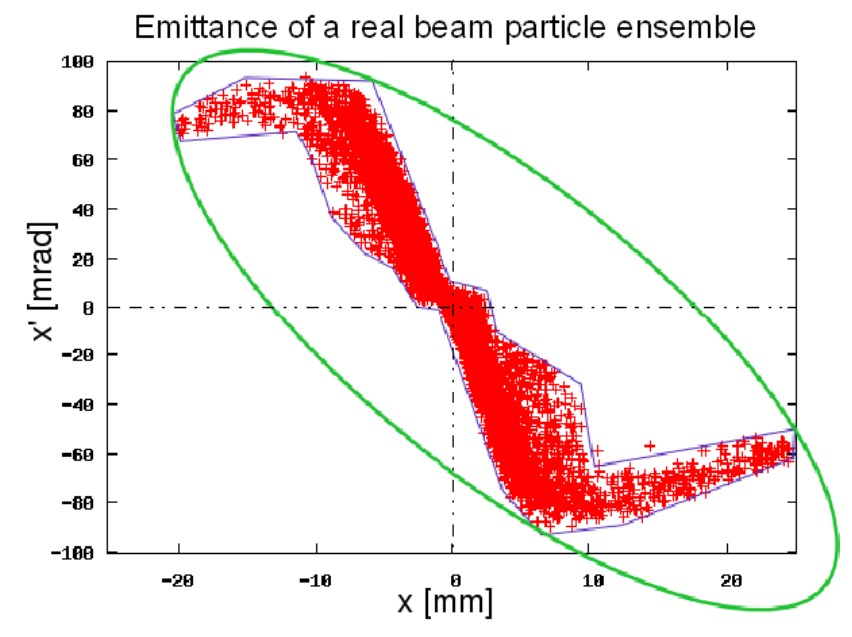

Fig. 1. 2-dimensional phase space plot showing a phase space distribution (red) acquired by an emittance scanner and the corresponding emittance (green).

\subsection{Ion beams}

\subsubsection{Characterization of ion beams}

Beam particles form an ensemble, whose energy can be described using a hamilton function. It is by definition translation invariant, time invariant and rotation invariant. Noether's theorem states that these invariances lead to corresponding conversation laws, therefore limiting the movement of the particles within its 6-dimensional phase space. Analogue to the movement of a particle within an incompressible liquid, beam particles moving along a linear path - the beam axis - follow elliptic paths within the phase space. Therefore, the phase space inhabitet by an ideal beam particle ensemble is an ellipsoid. It can easily be plotted by mapping the difference of the position of a particle in a dimension perpendicular to the beam axis ( $\mathrm{x}$-direction) to its first derivative. The area of this ellipse is called emittance of the beam. It is often used as a measure of beam quality.

The phase space inhabited by a beam can be measured using so-called phase space scanners (Strehl, 1987). It usually does not display an eliptic shape. In this case, the emittance is defined as the area of the smallest ellipse that includes all points of the phase space measured as shown in Fig. 1

\subsubsection{Non destructive beam surveillance}

Non destructive beam diagnostics usually are based on the output of optical sensors, e.g. CCD cameras that acquire light intensity distributions perpendicular to the beam axis. From these images, iso levels of constant intensity and the beam axis can be extracted. In this paper, we focus on extracting the main axis of the emittance ellipse by calculating points $\left(x, x^{\prime}\right)$ from a fixed position along the beam axis. The main axis of the ellipse can be used to evaluate the position of the phase space ellipse. This can be a direct measure for the beam quality, as the angle between the main axis of the emittance ellipse and the $x$-axis should increase if the beam is more focussed. Also, a shifting of the beam can be detected as a shifting of the main axis of the emittance ellipse.

\section{Algorithm}

The general strategy of estimating the orientation of the principal axis of the phase space ellipse is to extract iso levels of same intensity and then calculate points on the main axis of the phase space ellipse by measuring the distance $x$ between the beam axis and the iso level and the angle $x^{\prime}$ between the iso level and the beam axis.

All algorithms to be developed within our project have been implemented on the EyeRIS v1.1 system with special regard to computation time. Therefore, special attention had to be payed to which kind of CNN operations to be used for the extraction of iso levels. The images used for the development and test of algorithms were downsampled to the resolution of the EyeRIS v1.1 System of $128 \times 128$ pixels. The central task of calculating beam parameters is the extraction of iso levels with constant light intensity from images aquired by optical sensors.

\subsection{Extraction of iso levels}

The iso levels to be extracted can be described by contours of the binarized original image. Firstly, image quality has to be enhanced and contours have to be smoothed in order to determine beam parameters. A flow diagram of the iso level extraction algorithm can be found in Fig. 2.

First of all, the original picture is scaled to the range of $[-1 ; 1]$ and binarized by a threshold operation (CSWC Lab, 2007). The threshold has to be chosen according to the intensity of the iso level to be extracted. It will vary throughout the process of determining the beam parameters. However, the edge of the beam silhouette representing the iso level is noisy and has to be smoothed prior to the computation of beam parameters.

The processing steps used for smoothing of the beam silhouette are shown in Fig. 2. Firstly, a diffusion operation is applied which can be carried out very efficiently using a resistance grid of the FPP. The diffusion operation will be paused after the minimum execution time of $71.43 \mathrm{~ns}$ and the obtained intermediate diffusion image is binarized with a threshold that renders only about $10 \%$ of grey values into black and the rest into white, leading to a smoothed and slightly larger silhouette. Afterwards, the diffusion operation is continued for another $71.43 \mathrm{~ns}$ and binarization leads to an image similar to the intermediate diffuion image. The two diffused and binarized images are then combined by a logic XOR operation resulting in the extraction of 1 to 2 pixel wide 


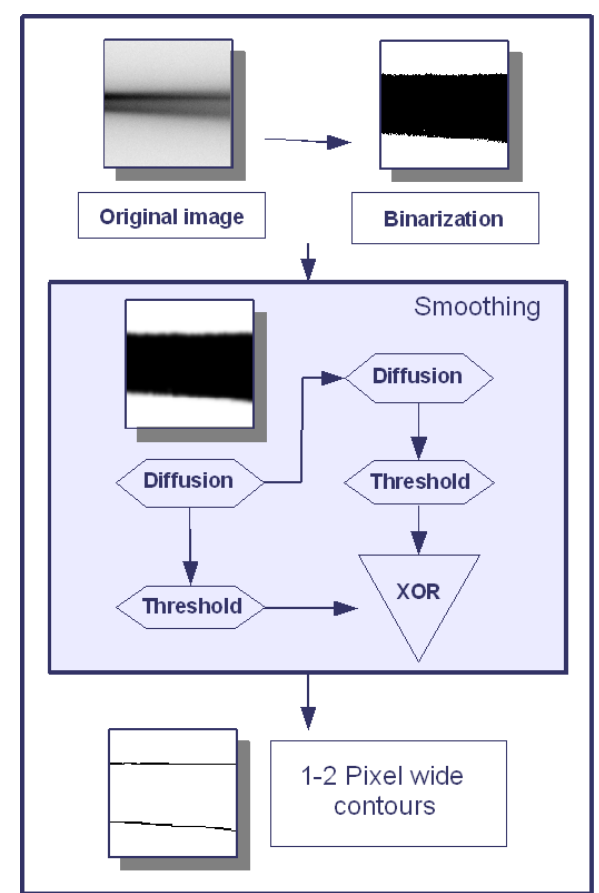

Fig. 2. Flow diagram of the developed algorithm used for the extraction of iso levels.
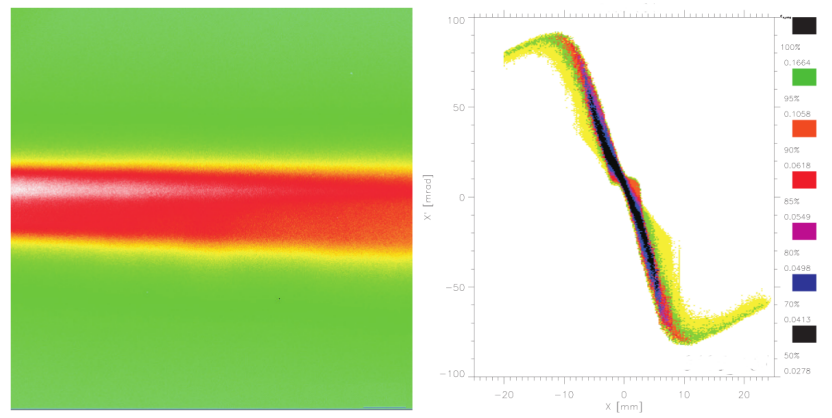

Fig. 3. Light intensity distributions (left) aquired for beam line configuration 1 and corresponding phase space representation (right).

contours corresponding to iso levels of constant light intensity. This process must be repeated for each intensity level to be included in the calculation of the phase space ellipse.

\section{Results}

In this paper we present the results for two different beam line configurations resulting from differently focussed beams with a deformation of the beam not detectable by conventional methods but visible in the image acquired for beam line configuration 2.

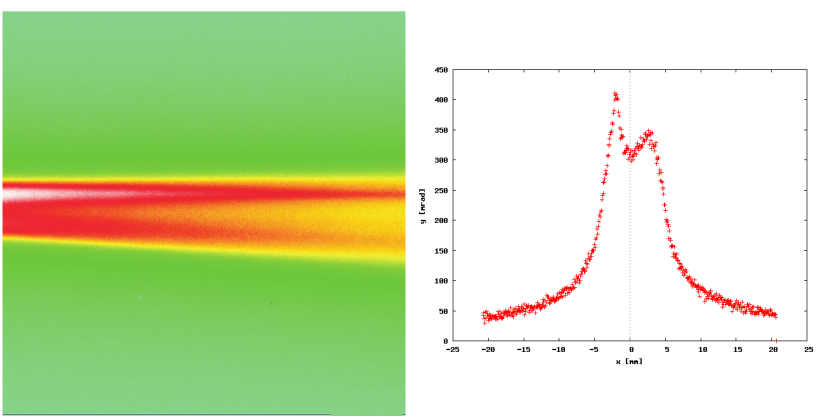

Fig. 4. Light intensity distribution (left) and intensity profile (right) aquired for beam line configuration 2 .

1. The first configuration is characterized by a focussed beam that is easily separable from the background as shown in Fig. 3. Its phase space distribution has a very characteristic shape of a diagonal line with two branches that form a $z$.

2. The second beam line configuration results in a beam picture very similar to the first one. However, close examination of the picture shows a deformation of the beam which is visible in the intensity profile of Fig. 4. This is not perceptable in the phase space distribution measured by conventional measurements.

\section{Estimated phase space distributions}

The estimation of the principal axis of the beam for configuration 1 is shown in Fig. 5. The calculated line is roughly parallel to the principal axis of the main branch of the phase space distribution measured by conventional methods (grey).

This result is different for beam line configuration 2 . In this case, the line of phase space points obtained by using the algorithm proposed in our paper and plotted in Fig. 6 does not match the main ellipse of the distribution acquired by the phase space scanner.

Instead, the axis (green) in Fig. 6 connects the outer ends of the determined distribution's side arms leading to a decrease of the angle between the the $\mathrm{x}$ - axis and the estimated main axis of the phase space distribution. The deflection of the beam leads to reduced focussing and therefore a change of shape of the phase space ellipse.

\subsection{Discussion}

Our results show that a deeper analysis of beam characteristics can be performed by applying CNN based strategies as compared to conventional methods. Beam dynamics can be determined in real-time without stopping ongoing experiments along the beam line. In this case, as in publications such as (Gabor, 2007), the results of optical methods for the 


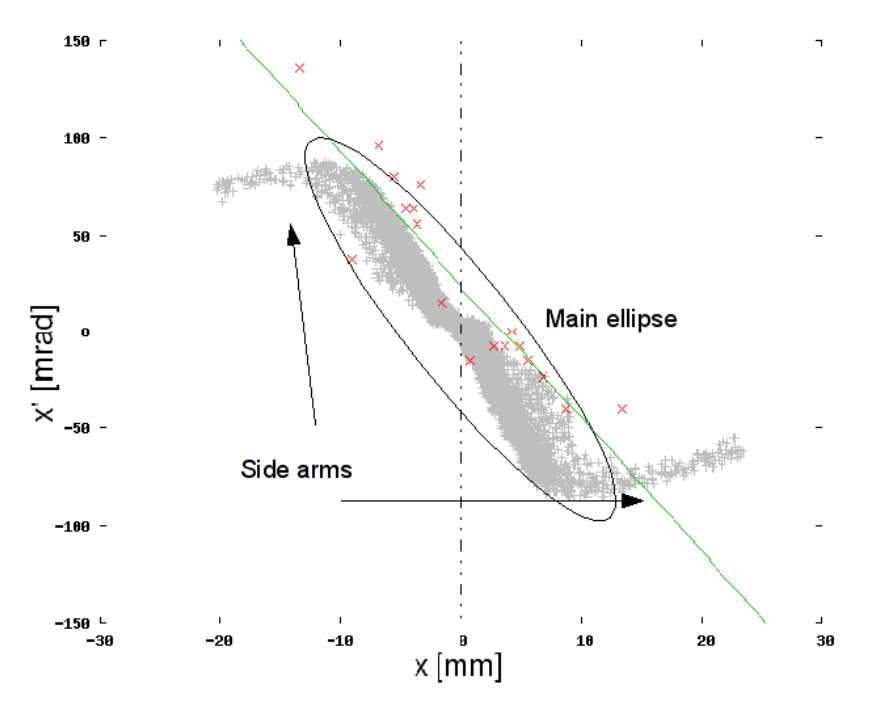

Fig. 5. Phase space points calculated by the application of our algorithm (red), estimated principal axis (green) and phase space distribution (grey) acquired by a phase space scanner for beam line configuration 1 . The estimated axis follows the main ellipse of the distribution acquired by a phase space scanner.

analysis of ion beams differ from conventional results, leading to a more correct evaluation of the beam.

\section{Conclusions}

In this paper we presented an algorithm for the CNNbased extraction of iso levels from optically aquired light density distributions. We showed that our algorithm is not only able to estimate correctly the orientation of the phase space distribution of beams but also to detect changes in beam quality for images with low resolutions. Thus, the use of hardware realizations of CNN could enable the development of powerful online beam control for the surveillance of particle beams parallel to ongoing experiments.

Acknowledgements. The authors would like to thank O. Meusel for supplying the data.

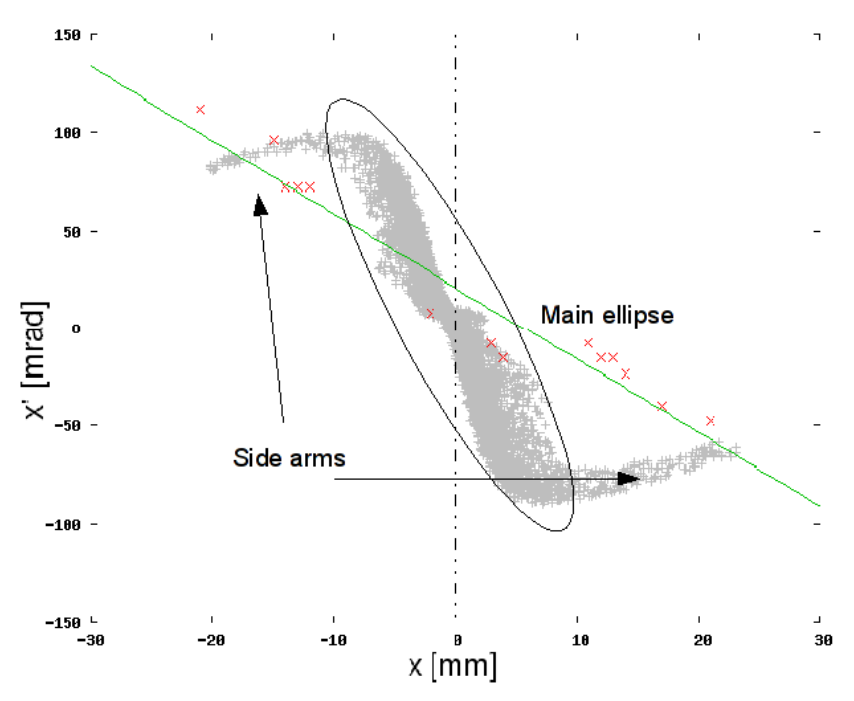

Fig. 6. Phase space points calculated by the application of our algorithm (red), estimated principal axis (green) and phase space distribution (grey) acquired by a phase space scanner for beam line configuration 2. The estimated axis does not follow the main ellipse but connects the side arms of the distribution.

\section{References}

Chua, L. O. and Yang, L.:Cellular Neural Networks: theory, IEEE T CIRCUITS SYST, 35, pp 1257-1272, Oktober 1988.

Chua, L. O and Roska, T.: CNN: A Paradigm for Complexity Volume 31 of World Scientific Series on Nonlinear Science, World Scientific Publishing Company, 1998

Gabor, C.: Untersuchungen zur zerstörungsfreien Emittanzmessung an einem negativen Wasserstoffionenstrahl, $\mathrm{PhD}$ thesis, JohannWolfgang- von Goethe- Universität, 2007.

Cellular Sensory Wave Computers Laboratory: Cellular Wave Computing Library, Budapest, Ungarn, 2007.

Chua, L. O. and Roska, T.: Cellular Neural Networks with Nonlinear and Delay-Type Template Elements and Non-Uniform Grids, International Journal on Circuit theory and Applications, 20,pp $469-481,1992$.

Anafocus Ldt.: Eye-RIS Vision System Hardware Description, Sevilla, Spain, 2005.

Nagy, Z. and Szolgay, P.: Configurable Multi-Layer CNN-UM Emulator on FPGA, IEEE Transactions on Circuits and Systems I: Fundamental Theory and Applications, 50, pp 774 - 778, 2003.

Strehl, P.: Beam diagnostics, Proc. of CAS, CERN accelerator school, CERN 1987. 